\title{
Neuer AG-Vorsitz Mammadiagnostik: eine volle Agenda
}

Prof. Dr. Markus Müller-Schimpfle, Chefarzt der Radiologie, Neuroradiologie und Nuklearmedizin am Klinikum Frankfurt-Höchst, und Partner der überörtlichen Gemeinschaftspraxis für Radiologie und Nuklearmedizin radiomedicum, ist neuer Leiter der AG Mammadiagnostik der Deutschen Röntgengesellschaft. Hier skizziert der Mammaspezialist die Herausforderung, die er für seine Amtszeit sieht.

\section{Working-Group Breast Imaging (WOBI) - Damit die Diskussionen dort geführt werden, wo sie hin- gehören \\ $\nabla$}

Viele Akteure, viele Modalitäten, eine hochkomplexe medizinische Materie und eine sensible Öffentlichkeit: die Brustkrebsdiagnostik ist in vielerlei Hinsicht ein heiß umkämpftes Feld. „Ich erinnere nur an die Statements der Schweizer Kollegen zum Mammografiescreening. Es gibt in den verschiedenen Fachgebieten, Sektoren, im deutschsprachigen Raum wie auch international scheinbar vollkommen unterschiedliche Ansätze und Betrachtungsweisen, die offen verlautbart werden. Mit dem Ergebnis, dass die Patientinnen und Screening-Teilnehmerinnen verwirrt werden und im schlimmsten Fall die Mammadiagnostik und Früherkennung komplett ablehnen und damit das Kind mit dem Bade ausschütten“, so Müller-Schimpfle.

Abhilfe schaffen könnte aus seiner Sicht die Etablierung einer Working-Group on Breast Imaging kurz WOBI. „Das darf am Anfang gerne eine Mailing-Group sein, in der man sich schnell und formlos über aktuelle Fragen austauscht." Wichtig ist dem Frankfurter Spezialisten, dass die fachbezogenen Diskussionen nicht in der breiten Öffentlichkeit stattfinden, sondern dort, wo sie hingehören: in der wissenschaftlichen Community. Das Ziel einer funktionierenden Working Group sieht MüllerSchimpfle in einer stärkeren Standardisierung und einer einvernehmlichen Sprechweise, die die Sicherheit der Betroffenen steigern und damit auch die Akzeptanz der Mammadiagnostik erhöhen hilft.

\section{Lernfähig und vertrauenswürdig: die Konsensustreffen}

Die Working Group ist geistesverwandt mit dem alle 2 Jahre stattfindenden und von Müller-Schimpfle initiierten Konsen- sustreffen. „Die Idee dieser Treffen ist es, die Fragen, die sich jetzt stellen, aber noch keine ausreichende Datengrundlage haben, durch kluge Köpfe zu erörtern. Wir wollen gemeinsam zu einer tragfähigen Mehrheitsmeinung gelangen, die so weit wie möglich vertrauenswürdig ist."

Wie wichtig der intensive Austausch in der senologischen Diagnostik ist, zeigt sich für Müller-Schimpfle aktuell an der Modalitätenfrage für Hochrisikopatientinnen: „2011 haben wir noch formuliert, die Röntgen-Mammografie und der Ultraschall sind die Grundlagen für diese Patientinnengruppe und die MRT die Ergänzung, 2013 hat unsere Diskussion ergeben, dass wir mittlerweile die MRT als primäre Modalität für Hochrisikopatientinnen und die beiden anderen Verfahren ergänzend sehen." Perspektivisch sieht der AG-Leiter die WOBI als Fortsetzung und Institutionalisierung der punktuellen Konsensustreffen.

\section{BI-RADS - Emanzipation vom ACR $\nabla$}

Eine Diskussion will Müller-Schimpfle auch für das BI-RADS anstoßen, dem Klassifikationslexikon des American College of Radiology (ACR). „Wir bekommen aus amerikanischer Sicht einen Standard aufgelegt, der nur eine gewisse Detailtiefe abbildet. Viele Fragen bleiben unbeantwortet, vieles darf mit gleicher medizinischer Berechtigung auch anders gesehen werden“, meint Müller-Schimpfle. Als Beispiel nennt er das Problem der Brustdichte. 2003 statuierte das BI-RADS-Lexikon die prozentuale Dichteangabe des Drüsengewebes, im gleichen Jahr hat seine damalige Tübinger Arbeitsgruppe festgestellt, dass es mehr Sinn mache und reproduzierbarer sei, auch die mögliche übersehene Herdgröße zu berücksichtigen. Im neuesten BI-RADS-Lexikon wird nun empfohlen, sich auch an der möglicherweise übersehenen Tumorgröße für die Parenchymklassen A-D zu orientieren,

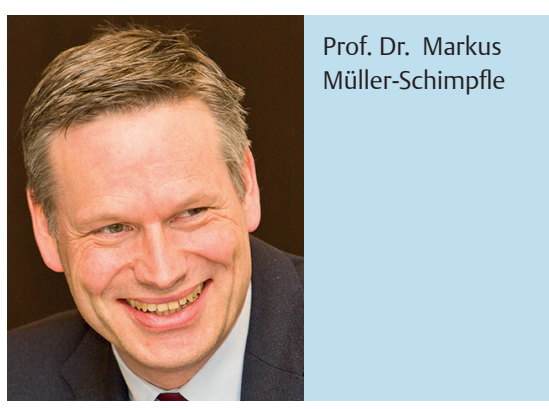

ohne sich aber konsequent zu spezifizieren. „BI-RADS ist eine gute Basis, aber wir müssen uns nicht jeder US-amerikanischen Empfehlung unterwerfen. Auch der deutschsprachige Wissenschaftsraum kann die medizinische Vergleichbarkeit gewährleisten.“

Also ein eigenes Lexikon? „Nicht kurzfristig“, meint Müller-Schimpfle. „Aber wir sollten das Thema in unserer deutschsprachigen Community sondieren." Sprechen möchte der neue AG-Vorsitzende mit dem ACR wegen der strikten und die wissenschaftliche Freiheit beschränkenden Copyright-Regelungen. „Hier dürfen wir uns nicht in Ketten legen lassen." Selbstverständlich sollte aber die wissenschaftliche Übertragbarkeit und damit Vergleichbarkeit von Befundungsstandards gewährleistet sein.

\section{Politische Intervention: die Radio- logie in der ASV \\ $\nabla$}

Müller-Schimpfle, der sich seit Jahren auch in der Berufs- und Standespolitik engagiert, steht aktuell in der Debatte um die ambulante spezialfachärztliche Versorgung (ASV). Das neue, sektorenübergreifende Kooperationsmodell wird zurzeit im Gemeinsamen Bundesausschuss (G-BA) anhand einzelner Krankheitsbilder konkretisiert.

Hauptproblem, das Müller-Schimpfle gemeinsam mit anderen Vertretern der DRG sieht, ist die Reduzierung des Radiologen auf den Auftragsnehmer für die Diagnostik. „Wir sind nur hinzuziehende Fachrichtung, wir können keine Teams bilden, wir werden nicht in die Verlaufskontrolle eingebunden." Höchst problematisch sieht er die ASV-Behandlung von Patienten mit fortgeschrittenen Gastrointestinal-Tumoren, in der die 24-Stunden-Erreichbarkeit eines Radiologen nicht vorgesehen ist. „Das könnte sich zu einem Knock-Out-Kriterium für das gesamte Vorhaben entwicklen“. 